\title{
ASYMPTOTICS OF WEIGHTED EMPIRICAL PROCESSES OF LINEAR FIELDS WITH LONG-RANGE DEPENDENCE
}

\author{
Paul DOUKHAN ${ }^{\mathrm{a}, *}$, Gabriel LANG $^{\mathrm{b}}$, Donatas SURGAILIS $^{\mathrm{c}}$ \\ ${ }^{a}$ LS-CREST and UPRESA 8088, Université Cergy Pontoise, 95302 Cergy-Pontoise cedex, France \\ ${ }^{\mathrm{b}}$ Laboratoire GRESE, ENGREF, 75732 Paris cedex 15, France \\ ${ }^{\mathrm{c}}$ Vilnius Institute of Mathematics and Informatics, 2600 Vilnius, Lithuania
}

Received 1 April 2001, revised 2 April 2002

ABSTRACT. - We discuss the asymptotic behavior of weighted empirical processes of stationary linear random fields in $\mathbb{Z}^{d}$ with long-range dependence. It is shown that an appropriately standardized empirical process converges weakly in the uniform-topology to a degenerated process of the form $f Z$, where $Z$ is a standard normal random variable and $f$ is the marginal probability density of the underlying random field.

(c) 2002 Éditions scientifiques et médicales Elsevier SAS

MSC: 60F17; 60G60

RÉSUMÉ. - Nous étudions le comportement asymptotique du processus empirique pondéré pour un champ linéaire stationnaire à longue dépendance sur $\mathbb{Z}^{d}$. Nous montrons que ce processus convenablement normalisé converge faiblement pour la topologie uniforme vers un processus dégénéré de la forme $f Z$, où $Z$ est une variable normale standard et $f$ est la densité de la marginale du champ considéré.

(c) 2002 Éditions scientifiques et médicales Elsevier SAS

\section{Introduction and main results}

Let $\left\{X_{t}, t \in \mathbb{Z}^{d}\right\}$ be a stationary ergodic random field indexed by points of the $d$ dimensional lattice $\mathbb{Z}^{d}$. The random field $\left\{X_{t}\right\}$ is said long-range dependent if its covariance function $r_{t}=\operatorname{cov}\left(X_{0}, X_{t}\right)$ is not summable: $\sum_{t \in \mathbb{Z}^{d}}\left|r_{t}\right|=\infty$, or if its spectral density is unbounded. Statistical analysis of long-range dependent random fields is important to many scientific areas, see Ivanov and Leonenko [11], Leonenko [16]. Limit theorems for sums of nonlinear functionals of long-range dependent Gaussian random fields were studied in Dobrushin and Major [5], Ivanov and Leonenko [11], Surgailis and Woyczynski [19], Leonenko and Woyczynski [17], Leonenko [16]. Marinucci [18] discussed estimation of the long memory parameter for Gaussian random fields with singular spectrum. See also Albeverio, Molchanov and Surgailis [1], Anh, Angulo and

\footnotetext{
* Corresponding author.

E-mail address: doukhan@ensae.fr (P. Doukhan).
} 
Ruiz-Medina [2] for other areas and problems related to random fields with long-range dependence.

The present paper studied the asymptotic behavior of the weighted empirical process

$$
\widehat{F}_{N}^{(\gamma, \xi)}(x)=N^{-d} \sum_{t \in A_{N}} \gamma_{N, t} I\left(X_{t} \leqslant x+\xi_{N, t}\right), \quad x \in \mathbb{R},
$$

of a long-range dependent random field observed on the "cube" $A_{N}=[1, N]^{d} \cap \mathbb{Z}^{d}$ and where the real coefficients $\left\{\xi_{N, t}, \gamma_{N, t} ; t \in A_{N}\right\}$ are non-random and uniformly bounded:

$$
\sup _{N>0} \max _{t \in A_{N}}\left(\left|\xi_{N, t}\right|+\left|\gamma_{N, t}\right|\right)=\mathrm{O}(1) .
$$

Weighted empirical processes arise in many statistical applications, see Koul [12], Koul and Mukherjee [13], and see also in Section 2. More precisely, we assume that $\left\{X_{t}\right\}$ is a linear random field:

$$
X_{t}=\sum_{u \in \mathbb{Z}^{d}} b_{u} \zeta_{t+u}, \quad t \in \mathbb{Z}^{d},
$$

where $\left\{\zeta_{u}, u \in \mathbb{Z}^{d}\right\}$ is an i.i.d. field with zero mean and variance 1 , and $\left\{b_{u}, u \in \mathbb{Z}^{d}\right\}$ are non-random weights which decay as $|u|^{-\beta}, \beta \in(d / 2, d)$. In the one-dimensional case $d=1$, this problem was studied by Dehling and Taqqu [3], Ho and Hsing [9], Giraitis, Koul and Surgailis [7], Koul and Surgailis [14] and others. Most of these works assume that $\left\{X_{t}\right\}$ is causal:

$$
X_{t}=\sum_{s \leqslant t} b_{t-s} \zeta_{s}, \quad t \in \mathbb{Z}
$$

The causality assumption lies at the basis of the martingale difference decomposition due to Ho and Hsing [9], which was also used in [10,14,6,8,15] to study asymptotic properties of nonlinear functionals of long memory moving averages (1.4). While causality is often assumed in time series, it appears rather artificial in the context of random fields $(d>1)$. Let us remark that the well-known approach via Hermite expansions (see e.g. [5,20,3]) does not apply to the present situation, as the random field (1.3) is not assumed to be Gaussian.

Let us formulate the main result of the paper. We shall assume that the weights $b_{u}$ are of the form

$$
b_{u}=B_{0}(u /|u|)|u|^{-\beta}, \quad u \in \mathbb{Z}^{d},
$$

where $d / 2<\beta<d$ and where $B_{0}(x), x \in S_{d-1}:=\left\{y \in \mathbb{R}^{d}:|y|=1\right\}$ is a bounded piece-wise continuous function. It is easy to verify that, under these assumptions, $r_{t}=\operatorname{cov}\left(X_{0}, X_{t}\right)=\left(R_{0}(t /|t|)+\mathrm{o}(1)\right)|t|^{d-2 \beta},|t| \rightarrow \infty$ and $\mathbb{E} \bar{X}_{N}^{2}=\left(c_{1}^{2}+\mathrm{o}(1)\right) N^{d-2 \beta}$, $N \rightarrow \infty$, where $\bar{X}_{N}=N^{-d} \sum_{t \in A_{N}} X_{t}$ is the sample mean, and $R_{0}(x)$ and $c_{1}$ is some continuous function on $S_{d-1}$ and a finite constant, respectively. The previous formulation (1.5) allows anisotropic behaviors of coefficients $\left\{b_{u}, u \in \mathbb{Z}^{d}\right\}$. This form can be made more general by multiplication by a slowly varying function $L(|u|)$ as in [5].

Write $F(x)=\mathbb{P}\left(X_{0} \leqslant x\right), G(x)=\mathbb{P}\left(\zeta_{0} \leqslant x\right)$ for the marginal distribution functions, and $\Rightarrow_{D(\overline{\mathbb{R}})}$ for weak convergence of random elements with values in the Skorohod 
space $D(\overline{\mathbb{R}})$ of cadlag functions on $\overline{\mathbb{R}}=[-\infty,+\infty]$ with the sup-topology. To compare asymptotic behaviours of the weighted empirical process and the sample mean, introduce:

$$
\mathcal{S}_{N}^{(\gamma, \xi)}(x)=\sum_{t \in A_{N}} \gamma_{N, t}\left\{I\left(X_{t} \leqslant x+\xi_{N, t}\right)-F\left(x+\xi_{N, t}\right)+f\left(x+\xi_{N, t}\right) X_{t}\right\},
$$

where $f(x)=F^{\prime}(x)$ is the marginal density.

THEOREM 1.1. - Assume, in addition to (1.2), (1.3), (1.5), that the distribution of $\zeta_{0}$ satisfies the two following conditions: there exists constants $C, \delta>0$ such that

$$
\left|\mathbb{E} \mathrm{e}^{\mathrm{i} a \zeta_{0}}\right| \leqslant C(1+|a|)^{-\delta}, \quad a \in \mathbb{R},
$$

and

$$
\mathbb{E}\left|\zeta_{0}\right|^{2+\delta}<\infty
$$

Then for any $\varepsilon>0$ there exists $C(\varepsilon)<\infty$ such that, for any $N \geqslant 1$

$$
\mathbb{P}\left(\sup _{x \in \mathbb{R}} N^{\beta-3 d / 2}\left|\mathcal{S}_{N}^{(\gamma, \xi)}(x)\right|>\varepsilon\right) \leqslant C(\varepsilon) N^{-\kappa / 2}, \quad \kappa=\min \left\{\beta-\frac{d}{2}, \beta d\right\} .
$$

In the special case $\gamma_{N, t} \equiv 1, \xi_{N, t} \equiv 0, \widehat{F}_{N}^{(1,0)}(x) \equiv \widehat{F}_{N}(x)=N^{-d} \sum_{t \in A_{N}} I\left(X_{t} \leqslant x\right)$ is the empirical distribution function, and we obtain

COROLlaRY 1.2. - Under the conditions of Theorem 1.1, for any $\varepsilon>0, N \geqslant 1$

$$
\mathbb{P}\left(\sup _{x \in \mathbb{R}} N^{\beta-d / 2}\left|\widehat{F}_{N}(x)-F(x)+f(x) \bar{X}_{N}\right|>\varepsilon\right) \leqslant C(\varepsilon) N^{-\kappa / 2} .
$$

The above corollary together with asymptotic normality of the sample mean $\bar{X}_{N}$ yield the following

COROLLARY 1.3. - Under the conditions of Theorem 1.1,

$$
N^{\beta-d / 2}\left(\widehat{F}_{N}(x)-F(x)\right) \Rightarrow_{D(\overline{\mathbb{R}})} c_{1} f(x) Z,
$$

where $Z \sim N(0,1)$ is standard normal variable.

Theorem 1.1 provides the uniform reduction principle for weighted empirical processes of long-range dependent linear field (1.3), which is similar to the reduction principle of Dehling and Taqqu [3] and its generalizations [9,7,14,15] in the case of one-dimensional time. Conditions (1.6) and (1.7) are rather weak restrictions on the smoothness of the distribution of $\zeta$ and its moments, c.f. [8], Theorem 1.1. Those conditions can certainly be relaxed, but this would provide additional technical problems.

The rest of the paper is organized as follows. Section 2 contains some applications of the above results to rank statistics and regression estimators. The remaining Sections 35 are given to the proof of Theorem 1.1, which follows from the well-known chaining 
argument of Dehling and Taqqu [3] and Lemma 1.4 below. For any function $g(x), x \in \mathbb{R}$, and any $x<y$, put $g(x, y)=g(y)-g(x)$.

LEMmA 1.4. - There exists a finite measure $\mu$ on the real line, such that for any $N \geqslant 1$ and any $x<y$

$$
\mathbb{E}\left(\mathcal{S}_{N}^{(\gamma, \xi)}(x, y)\right)^{2} \leqslant \mu(x, y) N^{3 d-2 \beta-\kappa} .
$$

Let us explain the main idea of the proof of Lemma 1.4. Its starting point is the telescoping identity (1.10) originating to Ho and Hsing [9] (a similar identity is also used in $[10,14,8,15])$. Write $\mathcal{S}_{N}^{(\gamma, \xi)}(x, y)=\sum_{t \in A_{N}} \gamma_{N, t} H_{t}\left(X_{t}\right)$, where the function $H_{t}(z) \equiv$ $H_{t}(z ; x, y), z \in \mathbb{R}$, is defined, for any $x<y, t \in \mathbb{Z}$, by

$$
H_{t}(z)=I\left(x+\xi_{N, t}<z \leqslant y+\xi_{N, t}\right)-F\left(x+\xi_{N, t}, y+\xi_{N, t}\right)+f\left(x+\xi_{N, t}, y+\xi_{N, t}\right) z .
$$

With each point $t \in \mathbb{Z}^{d}$, we can associate a decreasing filtration

$$
\mathcal{F}_{t, s}:=\sigma\left\{\zeta_{t+u}: u \succ s\right\}, \quad s \succeq 0,
$$

corresponding to a complete order relation $\prec$ on $\mathbb{Z}^{d}$. Let $s-1$ be the index just before $s$ in the enumeration of $\mathbb{Z}^{d}$, and let $\mathcal{F}_{t,-1}=\sigma\left\{\zeta_{s}: s \in \mathbb{Z}^{d}\right\}$ be the basic $\sigma$-field. Then

$$
H_{t}\left(X_{t}\right)=\sum_{s \succeq 0} U_{t, s}(x, y),
$$

where $U_{t, s}(x, y)=U_{t, s}(y)-U_{t, s}(x)$ and

$$
\begin{aligned}
U_{t, s}(x):= & \mathbb{P}\left(X_{t} \leqslant x+\xi_{N, t} \mid \mathcal{F}_{t, s-1}\right)-\mathbb{P}\left(X_{t} \leqslant x+\xi_{N, t} \mid \mathcal{F}_{t, s}\right) \\
& +f\left(x+\xi_{N, t}\right) b_{s} \zeta_{t+s} .
\end{aligned}
$$

The series (1.10) converges in $L^{2}$ by orthogonality; however, for different $t$, filtrations (1.9) are not monotone as in the causal case. In particular, for $t+s \neq t^{\prime}+s$, the terms $U_{t, s}(x, y)$ and $U_{t^{\prime}, s^{\prime}}(x, y)$ are not orthogonal. The problem of the evaluation of the corresponding cross-covariances arises, in particular, of the covariances $\operatorname{cov}\left(U_{t, 0}(x, y), U_{t^{\prime}, 0}(x, y)\right)$ involving indicators $I\left(x<X_{t} \leqslant y\right), I\left(x<X_{t^{\prime}} \leqslant y\right)$. This is achieved by proving some asymptotic bounds for the bivariate probability density of $\left(X_{t}, X_{t^{\prime}}\right)$ as $\left|t-t^{\prime}\right| \rightarrow \infty$ (see Section 5 for details).

\section{Some applications}

\subsection{Rank statistics}

Denker [4] considers the rank statistic

$$
\widehat{T}_{N}(h):=N^{-d} \sum_{t \in A_{N}} \gamma_{N, t} h\left(X_{t}, \frac{R_{N, t}}{N^{d}+1}\right) .
$$


Here, $R_{N, t}=\sum_{s \in A_{N}} I\left(X_{s} \leqslant X_{t}\right)$ is the rank of $X_{t}$ among $X_{s}, s \in A_{N}, h: \mathbb{R} \times[0,1] \rightarrow \mathbb{R}$ is a given kernel, and $\left\{\gamma_{N, t}: t \in A_{N}\right\}$ are deterministic coefficients (weights). In order to write (2.1) as an integral w.r.t. empirical processes, introduce the weighted empirical process.

$$
\widehat{F}_{N}^{(\gamma)}(x):=N^{-d} \sum_{t \in A_{N}} \gamma_{N, t} I\left(X_{t} \leqslant x\right) .
$$

We shall assume that $\left\{\gamma_{N, t}\right\}$ are uniformly bounded and normalized: $N^{-d} \sum_{t \in A_{N}} \gamma_{N, t}=$ 1, implying $F^{(\gamma)}(x)=E \widehat{F}_{N}^{(\gamma)}(x)=F(x)$. Then $\widehat{T}_{N}(h)=H\left(c_{N} \widehat{F}_{N}, \widehat{F}_{N}^{(\gamma)}\right)$, where $c_{N}=$ $N^{d} /\left(N^{d}+1\right)=1+\mathrm{O}\left(N^{-d}\right)$ and $H(u, v)=\int_{\mathbb{R}} h(x, u(x)) d v(x)$.

We shall assume that the functional $H(u, v)$ is well-defined on the space of pairs $(u, v)$ of cadlag functions on $\mathbb{R}$ with bounded variation. Put $T(h):=H\left(F, F^{(\gamma)}\right)=$ $\int_{\mathbb{R}} h(x, F(x)) d F(x)$. By assuming some regularity and differentiability conditions on the kernel $h$, the difference $\widehat{T}_{N}(h)-T(h)$ can be written as

$$
\begin{aligned}
\widehat{T}_{N}(h)-T(h)= & \int_{\mathbb{R}} h_{y}(x, F(x))\left(\widehat{F}_{N}(x)-F(x)\right) d F^{(\gamma)}(x) \\
& +\int_{\mathbb{R}} h(x, F(x)) d\left(\widehat{F}_{N}^{(\gamma)}(x)-F^{(\gamma)}(x)\right) \\
& +\mathrm{o}\left(\left\|\widehat{F}_{N}-F\right\|+\left\|\widehat{F}_{N}^{(\gamma)}-F^{(\gamma)}\right\|\right),
\end{aligned}
$$

where $\|\cdot\|$ is the uniform distance. By applying Corollary 1.2 , under suitable conditions on $h(x, y)$ and $h_{y}(x, y)=\partial h(x, y) / \partial y$, from the above equation one obtains

$$
N^{\beta-d / 2}\left(\widehat{T}_{N}(h)-T(h)\right)=N^{\beta-d / 2}\left(h_{1} \bar{X}_{N}+h_{2} \bar{X}_{N}^{(\gamma)}\right)+\mathrm{o}_{P}(1),
$$

where

$$
\begin{aligned}
& \bar{X}_{N}^{(\gamma)}:=N^{-d} \sum_{t \in A_{N}} \gamma_{N, t} X_{t}, \quad h_{1}=\int_{\mathbb{R}} h_{y}(x, F(x)) f^{2}(x) d x, \quad \text { and } \\
& h_{2}=\int_{\mathbb{R}} h(x, F(x)) f(x) d x .
\end{aligned}
$$

Therefore, the limit distribution of $\widehat{T}_{N}(h)$ for long-range dependent linear observations $\left\{X_{t}\right\}$ is the same as the limit distribution of the linear combination of weighted sample means on the r.h.s. of (2.2). Let $\Sigma_{N}$ be the covariance matrix of the vector $N^{\beta-d / 2}\left(\bar{X}_{N}, \bar{X}_{N}^{(\gamma)}\right)$ with values in $\mathbb{R}^{2}$. By linearity of $\left\{X_{t}\right\}$ and the uniform boundedness of $\left\{\gamma_{N, t}\right\}$, one has the convergence in distribution

$$
N^{\beta-d / 2}\left(\bar{X}_{N}, \bar{X}_{N}^{(\gamma)}\right) \Rightarrow N(0, \Sigma),
$$

provided the $\operatorname{limit}_{N \rightarrow \infty} \Sigma_{N}=\Sigma$ exists [7, Theorem 2]. Clearly, (2.2) and (2.3) imply the asymptotic normality of the rank statistic:

$$
N^{\beta-d / 2}\left(\widehat{T}_{N}-T(h)\right) \Rightarrow N\left(0,{ }^{t} h \Sigma h\right), \quad{ }^{t} h=\left(h_{1}, h_{2}\right),
$$

where we write ${ }^{t} h$ for the transposed vector of $h$. 


\subsection{Regression estimators}

Consider the linear regression model

$$
Y_{N, t}={ }^{t} v_{N, t} \theta+X_{t}, \quad t \in A_{N},
$$

where $\theta \in \mathbb{R}^{p}$ is an unknown vector parameter and ${ }^{t} v_{N, t}$ is the $i$ th row of the $N^{d} \times p$ nonsingular design matrix $V_{N}$. The least squares estimator of $\theta$ is defined as $\hat{\theta}_{l s}=\left({ }^{t} V_{N} V_{N}\right)^{-1} \sum_{t \in A_{N}} v_{N, t} Y_{N, t}$. Koul and Mukherjee [13] (see also [7,15]) discussed asymptotic behavior of certain classes of robust $M$ - and $R$-estimators of $\theta$ in the case $d=1$ and long-range dependent "errors" $\left\{X_{t}\right\}$. The $M$-estimator $\hat{\theta}_{M}$ is defined, for a given nondecreasing function $\psi$ on $\mathbb{R}$, by $\hat{\theta}_{M}:=\operatorname{argmin}\left\{\left|\mathcal{M}_{N}(\theta)\right|: \theta \in \mathbb{R}^{d}\right\}, \mathcal{M}_{N}(\theta):=$ $\sum_{t \in A_{N}} v_{N, t} \psi\left(Y_{N, t}-{ }^{t} v_{N, t} \theta\right)$. The Jaeckel's rank estimator $\hat{\theta}_{J}$ is defined, for a given nondecreasing function $\phi$ on $(0,1)$, by $\hat{\theta}_{J}:=\operatorname{argmin}\left\{\left|\mathcal{J}_{N}(\theta)\right|: \theta \in \mathbb{R}^{d}\right\}, \mathcal{J}_{N}(\theta):=$ $\sum_{t \in A_{N}} v_{N, t} \phi\left(R_{N, t}(\theta) /\left(N^{d}+1\right)\right)$, where $R_{N, t}(\theta)$ denotes the rank of $Y_{N, t}-{ }^{t} v_{N, t} \theta$ among $Y_{N, s}-{ }^{t} v_{N, s} \theta, s \in A_{N}$. The classes of $M$ - and $R$-estimators are very large, in particular, the $M$-estimator corresponding to $\psi(x)=\operatorname{sgn}(x)$ is the least absolute deviation estimator, or the analog of the median in the location case.

As shown in Koul and Mukherjee [13], asymptotic properties of estimators $\hat{\theta}_{M}$ and $\hat{\theta}_{J}$ can be derived from asymptotic linearity of empirical processes $\mathcal{M}_{N}(\theta)$ and $\mathcal{J}_{N}(\theta)$, whose proof is based on the uniform reduction principle for weighted empirical process as in Theorem 1.1. Let $\theta_{0}$ be the true value of the regression parameter, then $N^{\beta-d / 2}\left(\hat{\theta}_{N}-\right.$ $\left.\theta_{0}\right)$ is a minimizer of $\left|N^{-d} \mathcal{M}_{N}\left(\theta_{0}+N^{d / 2-\beta} u\right)\right|$ with respect to $u \in \mathbb{R}^{d}$, where

$$
N^{-d} \mathcal{M}_{N}\left(\theta_{0}+N^{d / 2-\beta} u\right)=\int_{\mathbb{R}} \psi(x) d \widehat{F}_{N}^{(\gamma, \xi)}(x)
$$

is expressed via the weighted empirical process (1.1) corresponding to $\gamma_{N, t}=v_{N, t}$ and $\xi_{N, t}=N^{d / 2-\beta t} v_{N, t} u$. Using [13] and Theorem 1.1, exactly as in [7], Corollaries 1 and 3, one can obtain the representations

$$
\begin{aligned}
& N^{\beta-d / 2}\left(\hat{\theta}_{M}-\theta_{0}\right)=N^{\beta-d / 2}\left({ }^{t} V_{N} V_{N}\right)^{-1} \sum_{t \in A_{N}} v_{N, t} X_{t}+\mathrm{o}_{P}(1), \\
& N^{\beta-d / 2}\left(\hat{\theta}_{J}-\theta_{0}\right)=N^{\beta-d / 2}\left({ }^{t} \widetilde{V}_{N} \widetilde{V}_{N}\right)^{-1} \sum_{t \in A_{N}} \tilde{v}_{N, t} X_{t}+\mathrm{o}_{P}(1),
\end{aligned}
$$

where $\widetilde{V}_{N}$ is the $N^{d} \times p$ matrix of centered designs, with $i$ th row ${ }^{t} \tilde{v}_{N, t}={ }^{t} v_{N, t}-{ }^{t} \bar{v}_{N}$; $\bar{v}_{N}=N^{-d} \sum_{t \in A_{N}} v_{N, t}$. From the above relations, the asymptotic normality of the estimators $\hat{\theta}_{M}$ and $\hat{\theta}_{J}$, and their equivalence to the least squares estimator follow easily, see $[13,7]$.

\section{Proof of Lemma 1.4}

Let us introduce some notation. Let $|s|=\max _{1 \leqslant i \leqslant d}\left|s_{i}\right|, s=\left(s_{1}, \ldots, s_{d}\right) \in \mathbb{Z}^{d}$, be the sup norm on $\mathbb{Z}^{d}$. Let $0=s(0), s(1), s(2), \ldots$ be a complete enumeration of $\mathbb{Z}^{d}$ starting from the origin and filling the balls of $\mathbb{Z}^{d}$ following an increasing order; that is, if $s$ 
precedes $t$ in the enumeration, then $|s| \leqslant|t|$. From now on, consider that points of $\mathbb{Z}^{d}$ are ordered by this enumeration. The notations $\prec$ and $\succ$ correspond to this order; we write $s \preceq t$ (respectively, $s \succeq t$ ) if either $s \prec t$ or $s=t$ (respectively, either $s \succ t$ or $s=t$ ) hold. With each $t \in \mathbb{Z}^{d}$ we associate the decreasing filtration $\left\{\mathcal{F}_{t, s}, s \succeq 0\right\}$ (1.9), with the property that $\bigcap_{s \succeq 0} \mathcal{F}_{t, s}$ is the trivial $\sigma$-field. Write $X_{t, s}:=\sum_{0 \leq u<s} b_{u} \zeta_{t+u}, Y_{t, s}:=$ $\sum_{u \succ s} b_{u} \zeta_{t+u}$, so that $X_{t}=X_{t, s}+b_{s} \zeta_{t+s}+Y_{t, s}$ and $Y_{t, s}$ is $\mathcal{F}_{t, s}$-measurable. Let $F_{s}(x):=$ $\mathbb{P}\left(X_{t, s} \leqslant x\right)$ be the probability distribution function. Write $f(x)=F^{\prime}(x), f_{s}(x)=F_{s}^{\prime}(x)$ for the corresponding densities provided they exist. Below, we denote by $C$ a generic constant which may change from line to line. Put $B_{s}:=\mathbb{E} Y_{t, s}^{2}=\sum_{u \succ s} b_{u}^{2}$. Lemma 3.1 below is completely analogous to Giraitis and Surgailis [8], Lemma 2.1.

LEMMA 3.1. - There exist $s_{1} \succ 0$ and a constant $C<\infty$ such that for any $s \succ s_{1}$, the distribution functions $F(x), F_{s}(x)$ are twice continuously differentiable and, moreover, for any $s \succ s_{1}, x<y,|x-y| \leqslant 1$,

$$
\begin{gathered}
\left|F^{\prime \prime}(x)\right|+\left|F_{s}^{\prime \prime}(x)\right| \leqslant C\left(1+x^{2}\right)^{-1}, \\
\left|F^{\prime \prime}(x, y)\right|+\left|F_{s}^{\prime \prime}(x, y)\right| \leqslant C|x-y|\left(1+x^{2}\right)^{-1}, \\
\left|F^{\prime \prime}(x)-F_{s}^{\prime \prime}(x)\right| \leqslant C B_{s}\left(1+x^{2}\right)^{-1} .
\end{gathered}
$$

For $t \in \mathbb{Z}^{d}$ consider the telescoping identity (1.10). By the definition (1.11), $\mathbb{E}\left[U_{t, s}(x) \mid\right.$ $\left.\mathcal{F}_{t, s}\right]=0, s \succeq 0$. Furthermore,

$$
\begin{aligned}
U_{t, s}(x)= & F_{s}\left(x+\xi_{N, t}-b_{s} \zeta_{t+s}-Y_{t, s}\right)-\int_{\mathbb{R}} F_{s}\left(x+\xi_{N, t}-b_{s} z-Y_{t, s}\right) d G(z) \\
& +f\left(x+\xi_{N, t}\right) b_{s} \zeta_{t+s} .
\end{aligned}
$$

For $t+s \neq t^{\prime}+s^{\prime}$, introduce

$$
Y_{t, s ; t^{\prime}, s^{\prime}}:=Y_{t, s}-b_{t^{\prime}+s^{\prime}-t} \zeta_{t^{\prime}+s^{\prime}} I\left(t^{\prime}+s^{\prime}-t \succ s\right)=\sum_{u \succ s: t+u \neq t^{\prime}+s^{\prime}} b_{u} \zeta_{t+u},
$$

and

$$
\begin{aligned}
U_{t, s ; t^{\prime}, s^{\prime}}(x):= & \mathbb{P}\left(X_{t, s ; t^{\prime}, s^{\prime}} \leqslant x+\xi_{N, t} \mid \mathcal{F}_{t, s-1}\right)-\mathbb{P}\left(X_{t, s ; t^{\prime}, s^{\prime}} \leqslant x+\xi_{N, t} \mid \mathcal{F}_{t, s}\right) \\
& +f\left(x+\xi_{N, t}\right) b_{s} \zeta_{t+s},
\end{aligned}
$$

where

Then

$$
X_{t, s ; t^{\prime}, s^{\prime}}:= \begin{cases}X_{t}-b_{t^{\prime}+s^{\prime}-t} \zeta_{t^{\prime}+s^{\prime}}, & \text { if } t^{\prime}+s^{\prime}-t \succ s, \\ X_{t}, & \text { otherwise. }\end{cases}
$$

$$
\begin{aligned}
U_{t, s ; t^{\prime}, s^{\prime}}(x)= & F_{s}\left(x+\xi_{N, t}-b_{s} \zeta_{t+s}-Y_{t, s, t^{\prime}, s^{\prime}}\right) \\
& -\int_{\mathbb{R}} F_{s}\left(x+\xi_{N, t}-b_{s} u-Y_{t, s, t^{\prime}, s^{\prime}}\right) d G(u)+f\left(x+\xi_{N, t}\right) b_{s} \zeta_{t+s} .
\end{aligned}
$$

LEMMA 3.2. - For any $t, s, t^{\prime}, s^{\prime}, t+s \neq t^{\prime}+s^{\prime}, U_{t, s ; t^{\prime}, s^{\prime}}(x)$ is $\mathcal{F}_{t^{\prime}+s^{\prime}, 0}\left(=\sigma\left\{\zeta_{u}: u \neq\right.\right.$ $\left.\left.t^{\prime}+s^{\prime}\right\}\right)$-measurable and

$$
\mathbb{E}\left[U_{t, s ; t^{\prime}, s^{\prime}}(x) \mid \mathcal{F}_{t+s, 0}\right]=0, \quad \mathbb{E}\left[U_{t, s}(x) \mid \mathcal{F}_{t+s, 0}\right]=0 .
$$


Moreover, for any $x, y \in \mathbb{R}, U_{t, s ; t^{\prime}, s^{\prime}}(x)$ is orthogonal to both $U_{t^{\prime}, s^{\prime}}(y)$ and $U_{t^{\prime}, s^{\prime} ; t, s}(y)$.

Proof. $-\mathcal{F}_{t^{\prime}+s^{\prime}, 0}$-measurability of $U_{t, s ; t^{\prime}, s^{\prime}}(x)$ follows from (3.5) and the fact that $\zeta_{t+s}$ and $Y_{t, s ; t^{\prime}, s^{\prime}}$ are $\mathcal{F}_{t^{\prime}+s^{\prime}, 0}$-measurable. The first relation in (3.7) follows from (3.6) and from $\mathbb{E}\left[\zeta_{t+s} \mid \mathcal{F}_{t+s, 0}\right]=0, \mathbb{E}\left[F_{s}\left(x+\xi_{N, t}-b_{s} \zeta_{t+s}-Y_{t, s ; t^{\prime}, s^{\prime}}\right) \mid \mathcal{F}_{t+s, 0}\right]=\int F_{s}\left(x+\xi_{N, t}-\right.$ $\left.b_{s} z-Y_{t, s ; t^{\prime}, s^{\prime}}\right) d G(u)$, and the second one follows similarly. The above facts imply $\mathbb{E}\left[U_{t, s ; t^{\prime}, s^{\prime}}(x) U_{t^{\prime}, s^{\prime}}(y)\right]=\mathbb{E}\left[U_{t, s ; t^{\prime}, s^{\prime}}(x) \mathbb{E}\left[U_{t^{\prime}, s^{\prime}}(y) \mid \mathcal{F}_{t^{\prime}+s^{\prime}, 0}\right]\right]=0$ and, similarly,

$$
\mathbb{E}\left[U_{t, s ; t^{\prime}, s^{\prime}}(x) U_{t^{\prime}, s^{\prime} ; t, s}(y)\right]=\mathbb{E}\left[U_{t, s ; t^{\prime}, s^{\prime}}(x) \mathbb{E}\left[U_{t^{\prime}, s^{\prime} ; t, s}(y) \mid \mathcal{F}_{t^{\prime}+s^{\prime}, 0}\right]\right]=0 .
$$

Proof of Lemma 1.4. - By (1.10),

$$
\mathbb{E}\left(\mathcal{S}_{N}^{(\gamma, \xi)}(x, y)\right)^{2}=\sum_{t, t^{\prime} \in A_{N}} \gamma_{N, t} \gamma_{N, t^{\prime}} \sum_{s, s^{\prime} \succeq 0} \operatorname{cov}\left(U_{t, s}(x, y), U_{t^{\prime}, s^{\prime}}(x, y)\right),
$$

where $U_{t, s}(x, y)=U_{t, s}(y)-U_{t, s}(x)$. In the rest of the proof, we shall drop $x, y$ to simplify the notation. According to Lemma 3.2,

$$
\operatorname{cov}\left(U_{t, s}, U_{t^{\prime}, s^{\prime}}\right)=\operatorname{cov}\left(U_{t, s}-U_{t, s ; t^{\prime}, s^{\prime}}, U_{t^{\prime}, s^{\prime}}-U_{t^{\prime}, s^{\prime} ; t, s}\right),
$$

provided $t+s \neq t^{\prime}+s^{\prime}$. Therefore, by (1.5), $\mathbb{E}\left(S_{N}^{\gamma, \xi)}\right)^{2} \leqslant C\left(\Sigma_{N, 1}+\Sigma_{N, 2}+\Sigma_{N, 3}\right)$, where

$$
\begin{aligned}
& \Sigma_{N, 1}=\sum_{t, t^{\prime} \in A_{N}} \sum_{s, s^{\prime} \succeq 0: t+s=t^{\prime}+s^{\prime}}\left|\operatorname{cov}\left(U_{t, s}, U_{t^{\prime}, s^{\prime}}\right)\right|, \\
& \Sigma_{N, 2}=\sum_{t, t^{\prime} \in A_{N}} \sum_{s, s^{\prime} \succeq s_{1}: t+s \neq t^{\prime}+s^{\prime}}\left|\operatorname{cov}\left(U_{t, s}-U_{t, s ; t^{\prime}, s^{\prime}}, U_{t^{\prime}, s^{\prime}}-U_{t^{\prime}, s^{\prime} ; t, s}\right)\right|, \\
& \Sigma_{N, 3}=\sum_{t, t^{\prime} \in A_{N}} \sum_{0 \leq s, s^{\prime} \leq s_{1}: t+s \neq t^{\prime}+s^{\prime}}\left|\operatorname{cov}\left(U_{t, s}-U_{t, s ; t^{\prime}, s^{\prime}}, U_{t^{\prime}, s^{\prime}}-U_{t^{\prime}, s^{\prime} ; t, s}\right)\right| .
\end{aligned}
$$

Put $|s|_{+}^{-\lambda}=|s|^{-\lambda} \vee 1\left(\lambda>0, s \in \mathbb{Z}^{d}\right)$. By Lemma 3.3 below,

$$
\begin{aligned}
\Sigma_{N, 1} & \leqslant \sum_{t, t^{\prime} \in A_{N}} \sum_{s, s^{\prime} \succ 0: t+s=t^{\prime}+s^{\prime}}\left(\mathbb{E} U_{t, s}^{2}\right)^{1 / 2}\left(\mathbb{E} U_{t^{\prime}, s^{\prime}}^{2}\right)^{1 / 2} \\
& \leqslant C \mu(x, y) \sum_{t, t^{\prime} \in A_{N}} \sum_{s \in \mathbb{Z}^{d}}|s|_{+}^{-\alpha / 2}\left|t+s-t^{\prime}\right|_{+}^{-\alpha / 2} \\
& \leqslant C \mu(x, y) \sum_{t, t^{\prime} \in A_{N}}\left|t-t^{\prime}\right|_{+}^{d-\alpha} \leqslant C \mu(x, y) N^{3 d-\alpha} .
\end{aligned}
$$

By Lemma 3.4,

$$
\begin{aligned}
\Sigma_{N, 2} & \leqslant \sum_{t, t^{\prime} \in A_{N}} \sum_{s, s^{\prime} \in \mathbb{Z}^{d}} \mathbb{E}^{1 / 2}\left(U_{t, s}-U_{t, s ; t^{\prime}, s^{\prime}}\right)^{2} \mathbb{E}^{1 / 2}\left(U_{t^{\prime}, s^{\prime}}-U_{t^{\prime}, s^{\prime} ; t, s}\right)^{2} \\
& \leqslant \mu(x, y) \sum_{t, t^{\prime} \in A_{N}} \sum_{s, s^{\prime} \in \mathbb{Z}^{d}}|s|_{+}^{-\beta}\left|t^{\prime}+s^{\prime}-t\right|_{+}^{-\beta}\left|s^{\prime}\right|_{+}^{-\beta}\left|t+s-t^{\prime}\right|_{+}^{-\beta} \\
& \leqslant C \mu(x, y) \sum_{t, t^{\prime} \in A_{N}}\left|t-t^{\prime}\right|_{+}^{2 d-4 \beta} \leqslant C \mu(x, y) N^{4 d-4 \beta} .
\end{aligned}
$$


Finally, from Lemma 3.5,

$$
\Sigma_{N, 3} \leqslant C \mu(x, y) \sum_{t, t^{\prime} \in A_{N}}\left|t^{\prime}-t\right|_{+}^{d-(2+\delta) \beta} \leqslant C \mu(x, y) N^{3 d-2 \beta-\beta \delta} .
$$

From (3.9)-(3.11), Lemma 1.4 follows with $\kappa=\min (2 \beta-d, \alpha-2 \beta, \beta \delta)>0$.

LEMMA 3.3. - There exist $\alpha>2 \beta$ and a finite measure $\mu$ on $\mathbb{R}$ such that for any $t, s \in \mathbb{Z}^{d}, x<y$

$$
\mathbb{E}\left(U_{t, s}(x, y)\right)^{2} \leqslant \mu(x, y)|s|_{+}^{-\alpha} .
$$

LEMMA 3.4. - There exists a finite measure $\mu$ on $\mathbb{R}$ such that for any $x<y$ and any $t, t^{\prime}, s, s^{\prime} \in \mathbb{Z}^{d}, s, s^{\prime} \succ s_{1}, t+s \neq t^{\prime}+s^{\prime}, x<y$

$$
\mathbb{E}\left(U_{t, s}(x, y)-U_{t, s ; t^{\prime}, s^{\prime}}(x, y)\right)^{2} \leqslant \mu(x, y)|s|_{+}^{-2 \beta}\left|t^{\prime}+s^{\prime}-t\right|_{+}^{-2 \beta} .
$$

LEMMA 3.5. - There exists a finite measure $\mu$ on $\mathbb{R}$ such that for any $x<y$ and any $t, t^{\prime}, s, s^{\prime} \in \mathbb{Z}^{d}, t+s \neq t^{\prime}+s^{\prime}, 0 \preceq s, s \preceq s_{1}$

$$
\begin{aligned}
& \left|\operatorname{cov}\left(U_{t, s}(x, y)-U_{t, s ; t^{\prime}, s^{\prime}}(x, y), U_{t^{\prime}, s^{\prime}}(x, y)-U_{t^{\prime}, s^{\prime} ; t, s}(x, y)\right)\right| \\
& \quad \leqslant \mu(x, y)\left|t^{\prime}-t\right|_{+}^{d-(2+\delta) \beta},
\end{aligned}
$$

where $\delta>0$ is the same as in (1.7).

\section{Proofs of Lemmas 3.3 and 3.4}

For $\gamma>1, x \in \mathbb{R}$, put

$$
h_{\gamma}(x):=(1+|x|)^{\gamma}, \quad \lambda_{\gamma}(x):= \begin{cases}|x|, & \text { if }|x|<1, \\ |x|^{\gamma}, & \text { if }|x| \geqslant 1 .\end{cases}
$$

Note that bounds (3.1)-(3.3) of Lemma 3.1 are valid with $\left(1+x^{2}\right)^{-1}$ replaced by $h_{\gamma}(x)$, for $1<\gamma \leqslant 2$. We shall need some properties of functions $h_{\gamma}$. Put $\mu_{\gamma}(x, y):=$ $\int_{x}^{y} h_{\gamma}(w) d w, x<y$.

Lemma 4.1 (Koul and Surgailis [15, Lemma 5.1]). - There exists a constant $C_{\gamma}$ depending only on $\gamma>1$, such that, for any $x, y \in \mathbb{R}$,

$$
\begin{aligned}
h_{\gamma}(x+y) & \leqslant h_{\gamma}(x)(1 \vee|y|)^{\gamma}, \\
\left|\int_{0}^{y} h_{\gamma}(x+w) d w\right| & \leqslant h_{\gamma}(x) \lambda_{\gamma}(y) .
\end{aligned}
$$

LEMMA 4.2. - Let $\phi(x), x \in \mathbb{R}$, be a real valued function such that for some $1<\gamma \leqslant$ $2,|\phi(x)| \leqslant C h_{\gamma}(x)$ and

$$
|\phi(x)-\phi(y)| \leqslant C|x-y| h_{\gamma}(x), \quad|x-y| \leqslant 1 .
$$


Then for any $x<y, u, v, z \in \mathbb{R}$

$$
\begin{aligned}
& \left|\int_{x}^{y}(\phi(\xi+v)-\phi(\xi)) d \xi\right| \leqslant C \mu_{\gamma}(x, y) \lambda_{\gamma}(v), \\
& \left|\int_{0}^{v} d w \int_{x}^{y}(\phi(\xi+w-z)-\phi(\xi-z)) d \xi\right| \leqslant C \mu_{\gamma}(x, y)|v|^{\gamma}(1 \vee|z|)^{\gamma}, \\
& \left|\int_{0}^{v} d w \int_{x}^{y}(\phi(\xi+w-u-z)-\phi(\xi+w-z)) d \xi\right| \\
& \leqslant C \mu_{\gamma}(x, y) \lambda_{\gamma}(u) \lambda_{\gamma}(v)(1 \vee|z|)^{\gamma} .
\end{aligned}
$$

Proof. - Consider (4.5). Let $|v| \leqslant 1$. Then by (4.3), the 1.h.s. of (4.5) does not exceed:

$$
C \int_{-|v|}^{|v|} w d w \int_{x}^{y} h_{\gamma}(\xi-z) d \xi \leqslant C \mu_{\gamma}(x, y) v^{2}(1 \vee|z|)^{\gamma} \leqslant C \mu_{\gamma}(x, y)|v|^{\gamma}(1 \vee|z|)^{\gamma}
$$

according to (4.1). Now let $|v| \geqslant 1$, then the 1.h.s. of (4.5) does not exceed:

$$
\begin{aligned}
& C \int_{-|v|}^{|v|} d w \int_{x}^{y}\left(h_{\gamma}(\xi+w-z)+h_{\gamma}(\xi-z)\right) d \xi \\
& \leqslant C(1 \vee|z|)^{\gamma} \int_{x}^{y} d \xi \int_{-|v|}^{|v|}\left(h_{\gamma}(\xi+w)+h_{\gamma}(\xi)\right) d w \\
& \quad \leqslant C(1 \vee|z|)^{\gamma} \mu_{\gamma}(x, y)\left(|v|^{\gamma}+|v|\right),
\end{aligned}
$$

according to (4.2). This proves (4.5). The proof of (4.4) and (4.6) is similar.

Proof of Lemma 3.3. - Consider first the case $s \succ s_{1}$, where $s_{1}$ was defined in Lemma 3.1. According to the definition, $U_{t, s}(x, y)=\sum_{i=1}^{3} V_{i}$, where

$$
\begin{aligned}
& V_{1}:=\int_{\mathbb{R}} d G(u) \int_{-b_{s} u}^{b_{s} \zeta_{t+s}} d z \int_{\tilde{x}}^{\tilde{y}}\left[f_{s}^{\prime}\left(w+z-Y_{t, s}\right)-f_{s}^{\prime}\left(w-Y_{t, s}\right)\right] d w, \\
& V_{2}:=b_{s} \zeta_{t+s} \int_{\tilde{x}}^{\tilde{y}}\left[f^{\prime}(z)-f^{\prime}\left(z-Y_{t, s}\right)\right] d z, \\
& V_{3}:=b_{s} \zeta_{t+s} \int_{\tilde{x}}^{\tilde{y}}\left[f^{\prime}\left(z-Y_{t, s}\right)-f_{s}^{\prime}\left(z-Y_{t, s}\right)\right] d z,
\end{aligned}
$$

and where $\tilde{x}:=x+\xi_{N, t}, \tilde{y}:=y+\xi_{N, t}$. Note $\mu_{\gamma}(\tilde{x}, \tilde{y}) \leqslant C \mu_{\gamma}(x, y)$ by (1.5) and (4.1), where the constant $C$ does not depend on $x, y, t, N$. Applying Lemma 4.2 (4.5) to $V_{1}$, we obtain on the one hand: 


$$
\begin{aligned}
\left|V_{1}\right| & \leqslant C \mu_{\gamma}(\tilde{x}, \tilde{y}) \int_{\mathbb{R}} d G(u)\left[\left|b_{s} u\right|^{\gamma}+\left|b_{s} \zeta_{t+s}\right|^{\gamma}\right]\left(1 \vee\left|Y_{t, s}\right|\right)^{\gamma} \\
& \leqslant C \mu_{\gamma}(x, y)\left|b_{s}\right|^{\gamma}\left(1+\left|\zeta_{t+s}\right|^{\gamma}\right)\left(1 \vee\left|Y_{t, s}\right|\right)^{\gamma} .
\end{aligned}
$$

On the other hand, the function $f_{s}^{\prime}$ is bounded by the integrable function $h_{\gamma}$ so that the integral over $w$ is bounded by a constant. Thus,

$$
\left|V_{1}\right| \leqslant C \int_{\mathbb{R}} d G(u)\left|b_{s}\right|\left(\left|\zeta_{s}\right|+|u|\right) \leqslant C\left|b_{s}\right|\left(1+\left|\zeta_{t+s}\right|\right)
$$

Taking $2<1+\gamma \leqslant 2+\delta$, from (4.10) and (4.11) we get

$$
\mathbb{E} V_{1}^{2} \leqslant C \mu_{\gamma}(x, y)\left|b_{s}\right|^{1+\gamma} \mathbb{E}\left(1+\left|\zeta_{t+s}\right|^{1+\gamma}\right) \mathbb{E}\left(1 \vee\left|Y_{t, s}\right|\right)^{\gamma} \leqslant C \mu_{\gamma}(x, y)\left|b_{s}\right|^{1+\gamma}
$$

Consider $V_{2}$. By Lemmas 3.1 and 4.2,

$$
\left|V_{2}\right| \leqslant C\left|b_{s} \zeta_{t+s}\right| \mu_{2}(\tilde{x}, \tilde{y}) \lambda_{2}\left(Y_{t, s}\right) \leqslant C\left|b_{s} \zeta_{t+s}\right| \mu_{2}(x, y) \lambda_{2}\left(Y_{t, s}\right)
$$

Combining this with the bound $\left|V_{2}\right| \leqslant C\left|b_{s} \zeta_{t+s}\right|$, we obtain

$$
\mathbb{E} V_{2}^{2} \leqslant C \mu_{2}(x, y) b_{s}^{2} \mathbb{E} \zeta_{t+s}^{2}\left(\mathbb{E}\left|Y_{t, s}\right|+\mathbb{E} Y_{t, s}^{2}\right) \leqslant C \mu_{2}(x, y) b_{s}^{2} B_{s}^{1 / 2} .
$$

A similar bound follows for $V_{3}$. Indeed, by Lemmas 3.1 (3.3) and 4.1,

$$
\left|V_{3}\right| \leqslant C\left|b_{s} \zeta_{t+s}\right| B_{s} \int_{\tilde{x}}^{\tilde{y}} h_{2}\left(z-Y_{t, s}\right) d z \leqslant C \mu_{2}(x, y)\left|b_{s} \zeta_{t+s}\right| B_{s}\left(1 \vee\left|Y_{t, s}\right|\right)^{2}
$$

Combining (4.14) with $\left|V_{3}\right| \leqslant C\left|b_{s} \zeta_{t+s}\right|$, one obtains

$$
\mathbb{E} V_{3}^{2} \leqslant C \mu_{2}(x, y) b_{s}^{2} B_{s}\left(1 \vee \mathbb{E} Y_{t, s}^{2}\right) \leqslant C \mu_{2}(x, y) b_{s}^{2} B_{s} .
$$

By the ball filling property of the enumeration of $\mathbb{Z}^{d}$,

$$
B_{s} \leqslant C \sum_{|u| \geqslant|s|} b_{s}^{2} \leqslant C \sum_{|u| \geqslant|s|}|u|^{-2 \beta} \leqslant C|s|^{d-2 \beta} .
$$

From (4.12)-(4.16) we obtain the bound (3.12) for $s \succ s_{1}$, with $\alpha=\min ((2+\delta) \beta, 3 \beta-$ $d / 2)>2 \beta$.

In order to prove (3.12), it remains to obtain the bound $\mathbb{E}\left(U_{t, s}(x, y)\right)^{2} \leqslant \mu(x, y)(0 \preceq$ $\left.s \preceq s_{1}\right)$.

We have

$$
\begin{aligned}
\mathbb{E}\left(U_{t, s}(x, y)\right)^{2} & \leqslant \mathbb{E}\left(\mathbb{P}\left(\tilde{x}<X_{t} \leqslant \tilde{y} \mid \mathcal{F}_{t, s-1}\right)+\mathbb{P}\left(\tilde{x}<X_{t} \leqslant \tilde{y} \mid \mathcal{F}_{t, s}\right)+\left|f(\tilde{x}, \tilde{y}) b_{s} \zeta_{t+s}\right|\right)^{2} \\
& \leqslant C(F(\tilde{x}, \tilde{y})+f(\tilde{x}, \tilde{y})) .
\end{aligned}
$$

Using $F(\tilde{x}, \tilde{y}) \leqslant F(x, y)+\int_{|z| \leqslant\left|\xi_{N, t}\right|} d z \int_{x}^{y}\left|f^{\prime}(z+u)\right| d u$ together with (1.5), (3.1), (4.1), one obtains the required bound, with $\mu(x, y)=C\left(F(x, y)+\mu_{2}(x, y)\right)$ a finite measure. Lemma 3.3 is proved. 
Proof of Lemma 3.4. - We only need to consider the case $t^{\prime}+s^{\prime}-t \succ s$. Write

$$
\begin{aligned}
W & :=U_{t, s}(x, y)-U_{t, s, t^{\prime}, s^{\prime}}(x, y) \\
& =\int_{\mathbb{R}} d G(u) \int_{0}^{b_{t^{\prime}-t+s^{\prime}} \zeta_{t^{\prime}+s^{\prime}}} d w \int_{\tilde{x}}^{\tilde{y}}\left(f_{s}^{\prime}\left(w+\eta-b_{s} u-Y_{t, s}\right)-f_{s}^{\prime}\left(w+\eta-b_{s} \zeta_{t+s}-Y_{t, s}\right)\right) d \eta .
\end{aligned}
$$

Splitting the last difference as $\left(f_{s}^{\prime}\left(w+\eta-b_{s} u-Y_{t, s}\right)-f_{s}^{\prime}\left(w+\eta-Y_{t, s}\right)\right)-\left(f_{s}^{\prime}(w+\right.$ $\left.\left.\eta-b_{s} \zeta_{t+s}-Y_{t, s}\right)-f_{s}^{\prime}\left(w+\eta-Y_{t, s}\right)\right)$ and using Lemma 4.2 (4.6) and Lemma 4.1 (4.1), one has

$$
\begin{aligned}
|W| & \leqslant C \mu_{\gamma}(\tilde{x}, \tilde{y}) \lambda_{\gamma}\left(b_{t^{\prime}-t+s^{\prime}} \zeta_{t^{\prime}+s^{\prime}}\right)\left(1 \vee\left|Y_{t, s}\right|\right)^{\gamma}\left(\lambda_{\gamma}\left(b_{s} \zeta_{t+s}\right)+\int_{\mathbb{R}} \lambda_{\gamma}\left(b_{s} u\right) d G(u)\right) \\
& \leqslant C \mu_{\gamma}(x, y)\left|b_{s} b_{t^{\prime}+s^{\prime}-t}\right| \lambda_{\gamma}\left(\zeta_{t^{\prime}+s^{\prime}}\right)\left(\lambda_{\gamma}\left(\zeta_{t+s}\right)+\mathbb{E} \lambda_{\gamma}\left(\zeta_{t+s}\right)\right) .
\end{aligned}
$$

For $2<2 \gamma \leqslant 2+\delta, \mathbb{E} \lambda_{\gamma}^{2}\left(\zeta_{0}\right)<\infty$ and we obtain

$$
\mathbb{E} W^{2} \leqslant C\left(\mu_{\gamma}(x, y)\right)^{2} b_{s}^{2} b_{t^{\prime}+s^{\prime}-t}^{2} \mathbb{E} \lambda_{\gamma}^{2}\left(\zeta_{t+s}\right) \mathbb{E} \lambda_{\gamma}^{2}\left(\zeta_{t^{\prime}+s^{\prime}}\right) \leqslant C \mu_{\gamma}(x, y) b_{s}^{2} b_{t^{\prime}+s^{\prime}-t}^{2}
$$

thereby proving (3.13) and the lemma.

\section{Proof of Lemma 3.5}

We shall discuss the case $t^{\prime}=s=s^{\prime}=0$ only. We have $\operatorname{cov}\left(U_{0,0}(x, y)-U_{0,0 ; t, 0}(x, y)\right.$, $\left.U_{t, 0}(x, y)-U_{t, 0 ; 0,0}\right)=\sum_{\ell=1}^{4} \tilde{\rho}_{\ell t}$, where

$$
\begin{aligned}
& \tilde{\rho}_{1 t}:=\mathbb{E}\left(I_{X_{0} \in\left(\tilde{x}_{0}, \tilde{y}_{0}\right]}-I_{X_{0 ; t} \in\left(\tilde{x}_{0}, \tilde{y}_{0}\right]}\right)\left(I_{X_{t} \in\left(\tilde{x}_{t}, \tilde{y}_{t}\right]}-I_{X_{t ; 0} \in\left(\tilde{x}_{t}, \tilde{y}_{t}\right]}\right), \\
& \tilde{\rho}_{2 t}:=\mathbb{E}\left(\left(I_{X_{0} \in\left(\tilde{x}_{0}, \tilde{y}_{0}\right]}-I_{X_{0 ; t} \in\left(\tilde{x}_{0}, \tilde{y}_{0}\right]}\right) \mathbb{E}\left(I_{X_{t} \in\left(\tilde{x}_{t}, \tilde{y}_{t}\right]}-I_{X_{t ; 0} \in\left(\tilde{x}_{t}, \tilde{y}_{t}\right]} \mid \mathcal{F}_{t}\right)\right), \\
& \tilde{\rho}_{3 t}:=\mathbb{E}\left(\mathbb{E}\left(I_{X_{0} \in\left(\tilde{x}_{0}, \tilde{y}_{0}\right]}-I_{X_{0 ; t} \in\left(\tilde{x}_{0}, \tilde{y}_{0}\right]} \mid \mathcal{F}_{0}\right)\left(I_{X_{t} \in\left(\tilde{x}_{t}, \tilde{y}_{t}\right]}-I_{X_{t ; 0} \in\left(\tilde{x}_{t}, \tilde{y}_{t}\right]}\right)\right), \\
& \tilde{\rho}_{4 t}:=\mathbb{E}\left(\mathbb{E}\left(I_{X_{0} \in\left(\tilde{x}_{0}, \tilde{y}_{0}\right]}-I_{X_{0 ; t} \in\left(\tilde{x}_{0}, \tilde{y}_{0}\right]} \mid \mathcal{F}_{0}\right) \mathbb{E}\left(I_{X_{t} \in\left(\tilde{x}_{t}, \tilde{y}_{t}\right]}-I_{X_{t ; 0} \in\left(\tilde{x}_{t}, \tilde{y}_{t}\right]} \mid \mathcal{F}_{t}\right)\right),
\end{aligned}
$$

and where we put $\tilde{x}_{t}:=x+\xi_{N, t}, \quad \tilde{y}_{t}:=y+\xi_{N, t}, \mathcal{F}_{t}:=\mathcal{F}_{t, 0}=\sigma\left\{\zeta_{s}: s \neq t\right\}$ and $X_{0 ; t}:=$ $X_{0,0 ; t^{\prime}, 0}=\sum_{s \neq t} b_{s} \zeta_{s}, X_{t ; 0}:=X_{t, 0 ; t^{\prime}, 0}=\sum_{s \neq-t} b_{s} \zeta_{t+s}$. Let $\rho_{\ell t} \equiv \rho_{\ell t}\left(x_{1}, y_{1} ; x_{2}, y_{2}\right), \ell=$ $1, \ldots, 4$, be defined as above, with $\tilde{x}_{0}, \tilde{y}_{0}, \tilde{x}_{t}, \tilde{y}_{t}$ replaced by $x_{1}, y_{1}, x_{2}, y_{2}$, respectively. With Lemma 4.1 in mind, it suffices to show that for some $\gamma>1$ and any $x_{i}<y_{i}, i=$ $1,2, t \in \mathbb{Z}^{d}$

$$
\left|\rho_{\ell t}\right| \leqslant C \mu_{\gamma}\left(x_{1}, y_{1}\right) \mu_{\gamma}\left(x_{2}, y_{2}\right)|t|_{+}^{d-(2+\delta) \beta}, \quad \ell=1, \ldots, 4
$$

The proof below is restricted to the case $\ell=1$, as the remaining cases $\ell=2,3,4$ can be treated similarly. Indeed, put $\widehat{X}_{0}:=\sum_{s \neq 0} b_{s} \zeta_{s}, \widehat{X}_{0 ; t}:=\sum_{s \neq 0, t} b_{s} \zeta_{s}, \widehat{X}_{t}:=$ $\sum_{s \neq 0} b_{s} \zeta_{t+s}, \widehat{X}_{t ; 0}:=\sum_{s \neq 0,-t} b_{s} \zeta_{t+s}$, then

$$
\rho_{4 t}=\int_{\mathbb{R}} \int_{\mathbb{R}} \hat{\rho}_{t}\left(x_{1}-b_{0} v_{1}, y_{1}-b_{0} v_{1} ; x_{2}-b_{0} v_{2}, y_{2}-b_{0} v_{2}\right) d G\left(v_{1}\right) d G\left(v_{2}\right),
$$


where $\hat{\rho}_{t}\left(x_{1}, y_{1} ; x_{2}, y_{2}\right):=\mathbb{E}\left(I_{\widehat{X}_{0} \in\left(x_{1}, y_{1}\right]}-I_{\widehat{X}_{0 ; t} \in\left(x_{1}, y_{1}\right]}\right)\left(I_{\widehat{X}_{t} \in\left(x_{2}, y_{2}\right]}-I_{\widehat{X}_{t ; 0} \in\left(x_{2}, y_{2}\right]}\right)$. Exactly similarly as (5.1) for $\ell=1$, one can show the bound

$$
\left|\hat{\rho}_{t}\left(x_{1}, y_{1} ; x_{2}, y_{2}\right)\right| \leqslant C \mu_{\gamma}\left(x_{1}, y_{1}\right) \mu_{\gamma}\left(x_{2}, y_{2}\right)|t|_{+}^{d-(2+\delta) \beta}
$$

Then from (5.2), (5.3) and Lemma 4.1, formula (4.1), one obtains

$$
\left|\rho_{4 t}\right| \leqslant C|t|_{+}^{d-(2+\delta) \beta} \prod_{i=1}^{2} \int_{\mathbb{R}} \int_{x_{i}}^{y_{i}} h_{\gamma}\left(u_{i}+b_{0} v_{i}\right) d u_{i} d G\left(v_{i}\right) \leqslant C|t|_{+}^{d-(2+\delta) \beta} \prod_{i=1}^{2} \mu_{\gamma}\left(x_{i}, y_{i}\right)
$$

or the bound (5.1) for $\ell=4$; the cases $\ell=2,3$ can be treated similarly.

Denote $f_{00, t}\left(z_{1}, z_{2}\right), f_{01, t}\left(z_{1}, z_{2}\right), f_{10, t}\left(z_{1}, z_{2}\right), f_{11, t}\left(z_{1}, z_{2}\right)$ the joint probability densities of vectors $\left(X_{0}, X_{t}\right),\left(X_{0}, X_{t ; 0}\right),\left(X_{0 ; t}, X_{t}\right),\left(X_{0 ; t}, X_{t ; 0}\right)$, respectively. Then $\rho_{1 t}=$ $\int_{x_{1}}^{y_{1}} \int_{x_{2}}^{y_{2}} \Psi_{t}\left(z_{1}, z_{2}\right) d z_{1} d z_{2}$, where $\Psi_{t}\left(z_{1}, z_{2}\right):=\sum_{j, k=0}^{1}(-1)^{j+k} f_{j k, t}\left(z_{1}, z_{2}\right), z_{1}, z_{2} \in \mathbb{R}$. Now, according to Lemma 5.1 below, the bound $\left(1+\left|z_{1}\right|\right)^{\gamma}\left(1+\left|z_{2}\right|\right)^{\gamma}\left|\Psi_{t}\left(z_{1}, z_{2}\right)\right| \leqslant$ $C t^{d-(2+\delta) \beta}$ with $\gamma=1+\delta^{\prime}>1$ follows from (5.5) if $\left|z_{1}\right| \leqslant c_{2},\left|z_{2}\right| \leqslant c_{2}$, from (5.7) if $\left|z_{1}\right|>c_{2},\left|z_{2}\right|>c_{2}$, and from (5.6) elsewhere. This entails (5.1) for $\ell=1$ and completes the proof of Lemma 3.5.

LEMMA 5.1. - There exists a locally bounded function $q(z), z \in \mathbb{R}$ satisfying

$$
|q(z)| \geqslant c_{1}|z|^{\delta^{\prime}}, \quad|z|>c_{2},
$$

where $c_{1}, c_{2}>0, \delta^{\prime}>0$ are some constants, such that for all $z_{1}, z_{2} \in \mathbb{R}^{2}$

$$
\begin{aligned}
\left|\Psi_{t}\left(z_{1}, z_{2}\right)\right| & \leqslant C|t|^{d-(2+\delta) \beta}, \\
\left|z_{i} q\left(z_{i}\right) \Psi_{t}\left(z_{1}, z_{2}\right)\right| & \leqslant C|t|^{d-(2+\delta) \beta}, \quad i=1,2, \\
\left|z_{1} z_{2} q\left(z_{1}\right) q\left(z_{2}\right) \Psi_{t}\left(z_{1}, z_{2}\right)\right| & \leqslant C|t|^{d-(2+\delta) \beta} .
\end{aligned}
$$

Proof. - Write $\Psi_{t}\left(z_{1}, z_{2}\right)=(2 \pi)^{-2} \int_{\mathbb{R}} \int_{\mathbb{R}} \mathrm{e}^{-\mathrm{i}\left(u_{1} z_{1}+u_{2} z_{2}\right)} \widehat{\Psi}_{t}\left(u_{1}, u_{2}\right) d u_{1} d u_{2}$, where the Fourier transform $\widehat{\Psi}_{t}\left(u_{1}, u_{2}\right)=\sum_{j, k=0}^{1}(-1)^{j+k} \hat{f}_{j k, t}\left(u_{1}, u_{2}\right)$, and

$$
\begin{array}{ll}
\hat{f}_{00, t}\left(u_{1}, u_{2}\right)=\mathbb{E} \mathrm{e}^{\mathrm{i}\left(u_{1} X_{0}+u_{2} X_{t}\right)}, & \hat{f}_{01, t}\left(u_{1}, u_{2}\right)=\mathbb{E} \mathrm{e}^{\mathrm{i}\left(u_{1} X_{0}+u_{2} X_{t ; 0}\right)}, \\
\hat{f}_{10, t}\left(u_{1}, u_{2}\right)=\mathbb{E} \mathrm{e}^{\mathrm{i}\left(u_{1} X_{0 ; t}+u_{2} X_{t}\right)}, & \hat{f}_{11, t}\left(u_{1}, u_{2}\right)=\mathbb{E} \mathrm{e}^{\mathrm{i}\left(u_{1} X_{0 ; t}+u_{2} X_{t ; 0}\right)}
\end{array}
$$

are the corresponding bivariate characteristic functions. Put $\phi(u):=\mathbb{E} \mathrm{e}^{\mathrm{i} u \zeta_{0}}, u \in \mathbb{R}$. Then

$$
\begin{aligned}
& \hat{f}_{00, t}\left(u_{1}, u_{2}\right)=\prod_{s} \phi\left(u_{1} b_{s}+u_{2} b_{s-t}\right), \\
& \hat{f}_{01, t}\left(u_{1}, u_{2}\right)=\phi\left(u_{1} b_{0}\right) \prod_{s \neq 0} \phi\left(u_{1} b_{s}+u_{2} b_{s-t}\right), \\
& \hat{f}_{10, t}\left(u_{1}, u_{2}\right)=\phi\left(u_{2} b_{0}\right) \prod_{s \neq t} \phi\left(u_{1} b_{s}+u_{2} b_{s-t}\right), \\
& \hat{f}_{11, t}\left(u_{1}, u_{2}\right)=\phi\left(u_{1} b_{0}\right) \phi\left(u_{2} b_{0}\right) \prod_{s \neq 0, t} \phi\left(u_{1} b_{s}+u_{2} b_{s-t}\right),
\end{aligned}
$$




$$
\begin{aligned}
\widehat{\Psi}_{t}\left(u_{1}, u_{2}\right)= & \left(\phi\left(u_{1} b_{0}+u_{2} b_{-t}\right)-\phi\left(u_{1} b_{0}\right)\right)\left(\phi\left(u_{1} b_{t}+u_{2} b_{0}\right)-\phi\left(u_{2} b_{0}\right)\right) \\
& \times \prod_{s \neq 0, t} \phi\left(u_{1} b_{s}+u_{2} b_{s-t}\right) .
\end{aligned}
$$

Using (1.6), similarly as in Giraitis, Koul and Surgailis [7, p. 323], one can show that for any $k \geqslant 0$ there exist $L>0$ and a constant $C<\infty$ such that for all $u_{1}, u_{2} \in \mathbb{R}$ and all $|t|>L$

$$
\left|\prod_{s \neq 0, t} \phi\left(u_{1} b_{s}+u_{2} b_{s-t}\right)\right| \leqslant C\left(1+\left|u_{1}\right|+\left|u_{2}\right|\right)^{-k} .
$$

By the inequality $\left|\phi\left(u_{1}+u_{2}\right)-\phi\left(u_{1}\right)\right| \leqslant\left|u_{2}\right| \mathbb{E}\left|\zeta_{0}\right|$, from (5.8), (5.9) one obtains

$$
\left|\widehat{\Psi}_{t}\left(u_{1}, u_{2}\right)\right| \leqslant C\left|b_{t} b_{-t}\right|\left|u_{1} u_{2}\right|\left(1+\left|u_{1}\right|+\left|u_{2}\right|\right)^{-k} .
$$

As $\int_{\mathbb{R}^{2}}\left|u_{1} u_{2}\right|\left(1+\left|u_{1}\right|+\left|u_{2}\right|\right)^{-k} d u_{1} d u_{2}<\infty$ for $k \geqslant 5$, this proves (5.5).

The proof of (5.6) is similar to that of (5.5) and (5.7) and we shall discuss the latter one only. Write

$$
z_{1} z_{2} \Psi_{t}\left(z_{1}, z_{2}\right)=-(2 \pi)^{-2} \int_{\mathbb{R}^{2}} \mathrm{e}^{-\mathrm{i}\left(z_{1} u_{1}+z_{2} u_{2}\right)} \partial_{12} \widehat{\Psi}_{t}\left(u_{1}, u_{2}\right) d u_{1} d u_{2},
$$

where $\partial_{12} \widehat{\Psi}_{t}\left(u_{1}, u_{2}\right)=\partial^{2} \widehat{\Psi}_{t}\left(u_{1}, u_{2}\right) / \partial u_{1} \partial u_{2}$. Further, let the function $q(z)$ be defined by

$$
\begin{aligned}
q(z) & =\int_{0}^{\infty}\left(1-\mathrm{e}^{-\mathrm{i} z \xi}\right) \xi^{-1-\delta^{\prime}} d \xi-\int_{1}^{\infty}\left(1-\mathrm{e}^{-\mathrm{i} z \xi}\right) \xi^{-1-\delta^{\prime}} d \xi \\
& =|z|^{\delta^{\prime}} \mathrm{e}^{\mathrm{i} \operatorname{sgn}(z) \pi \delta^{\prime} / 2} \pi / \sin \left(\pi \delta^{\prime}\right)-\tilde{q}(z),
\end{aligned}
$$

where $0<\delta^{\prime}<1$ and $\tilde{q}(z)=\int_{1}^{\infty}\left(1-\mathrm{e}^{-\mathrm{i} z \xi}\right) \xi^{-1-\delta^{\prime}} d \xi$ is bounded on $\mathbb{R}$. Therefore $q(z)$ satisfies (5.4). For any smooth, integrable test function $\psi$, let $\hat{\psi}$ be its Fourier transform, then:

$$
q(z) \psi(z)=(2 \pi)^{-1} \int_{\mathbb{R}} \mathrm{e}^{-\mathrm{i} z u} d u \int_{0}^{1}(\hat{\psi}(u)-\hat{\psi}(u-\xi)) \xi^{-1-\delta^{\prime}} d \xi .
$$

From (5.11) and (5.13), one infers

$$
\begin{aligned}
& q\left(z_{1}\right) q\left(z_{2}\right) z_{1} z_{2} \Psi_{t}\left(z_{1}, z_{2}\right) \\
& \quad=-(2 \pi)^{-2} \int_{\mathbb{R}^{2}} \mathrm{e}^{-\mathrm{i}\left(u_{1} z_{1}+u_{2} z_{2}\right)} d u_{1} d u_{2} \int_{[0,1]^{2}} \Delta_{\xi_{1} \xi_{2}} \partial_{12} \widehat{\Psi}_{t}\left(u_{1}, u_{2}\right) \xi_{1}^{-1-\delta^{\prime}} \xi_{2}^{-1-\delta^{\prime}} d \xi_{1} d \xi_{2},
\end{aligned}
$$

where $\Delta_{\xi_{1} \xi_{2}}$ is the double difference operator defined by $\Delta_{\xi_{1} \xi_{2}} \psi\left(u_{1}, u_{2}\right):=\psi\left(u_{1}, u_{2}\right)-$ $\psi\left(u_{1}-\xi_{1}, u_{2}\right)-\psi\left(u_{1}, u_{2}-\xi_{2}\right)+\psi\left(u_{1}-\xi_{1}, u_{2}-\xi_{2}\right), u_{1}, u_{2} \in \mathbb{R}, \xi_{1}, \xi_{2} \in[0,1]$. Consequently, 


$$
\begin{aligned}
& \left|q\left(z_{1}\right) q\left(z_{2}\right) z_{1} z_{2} \Psi_{t}\left(z_{1}, z_{2}\right)\right| \\
& \quad \leqslant C \int_{\mathbb{R}^{2}} d u_{1} d u_{2}\left|\int_{[0,1]^{2}} \Delta_{\xi_{1} \xi_{2}} \partial_{12} \widehat{\Psi}_{t}\left(u_{1}, u_{2}\right) \xi_{1}^{-1-\delta^{\prime}} \xi_{2}^{-1-\delta^{\prime}} d \xi_{1} d \xi_{2}\right| .
\end{aligned}
$$

To evaluate the r.h.s. of (5.14), it is convenient to rewrite the product (5.8) as $\widehat{\Psi}_{t}\left(u_{1}, u_{2}\right)=\prod_{s \succeq 0} \psi_{s}\left(u_{1}, u_{2}\right)$, where

$$
\psi_{s}\left(u_{1}, u_{2}\right):= \begin{cases}\phi\left(u_{1} b_{s}+u_{2} b_{s-t}\right), & \text { if } s \neq 0, s \neq t \\ \phi\left(u_{1} b_{0}+u_{2} b_{-t}\right)-\phi\left(u_{1} b_{0}\right), & \text { if } s=0 \\ \phi\left(u_{1} b_{t}+u_{2} b_{0}\right)-\phi\left(u_{2} b_{0}\right), & \text { if } s=t\end{cases}
$$

Then

$$
\partial_{12} \widehat{\Psi}_{t}=\sum_{\tau_{1}, \tau_{2} \succeq 0} \prod_{s \succeq 0} \vartheta_{\tau_{1} \tau_{2}} \psi_{s}
$$

where

$$
\vartheta_{\tau_{1} \tau_{2}} \psi_{s}:= \begin{cases}\partial_{12} \psi_{s}, & s=\tau_{1}=\tau_{2}, \\ \partial_{1} \psi_{s}, & s=\tau_{1} \neq \tau_{2}, \\ \partial_{2} \psi_{s}, & s=\tau_{2} \neq \tau_{1}, \\ \psi_{s}, & s \neq \tau_{1}, s \neq \tau_{2},\end{cases}
$$

and where $\partial_{j} \psi=\partial \psi / \partial u_{j}, j=1,2, \partial_{12} \psi=\partial^{2} \psi / \partial u_{1} \partial u_{2}$. Introduce also

$$
\theta_{\tau_{1} \tau_{2}}^{\xi_{1} \xi_{2}} \psi_{s}\left(u_{1}, u_{2}\right)= \begin{cases}\Delta_{\xi_{1} \xi_{2}} \psi_{s}\left(u_{1}, u_{2}\right), & s=\tau_{1}=\tau_{2}, \\ \psi_{s}\left(u_{1}, u_{2}\right)-\psi_{s}\left(u_{1}-\xi_{1}, u_{2}\right), & s=\tau_{1} \succ \tau_{2}, \\ \psi_{s}\left(u_{1}, u_{2}-\xi_{2}\right)-\psi_{s}\left(u_{1}-\xi_{1}, u_{2}-\xi_{2}\right), & s=\tau_{1} \prec \tau_{2}, \\ \psi_{s}\left(u_{1}, u_{2}\right)-\psi_{s}\left(u_{1}, u_{2}-\xi_{2}\right), & s=\tau_{2} \succ \tau_{1}, \\ \psi_{s}\left(u_{1}-\xi_{1}, u_{2}\right)-\psi_{s}\left(u_{1}-\xi_{1}, u_{2}-\xi_{2}\right), & s=\tau_{2} \prec \tau_{1}, \\ \psi_{s}\left(u_{1}-\xi_{1}, u_{2}-\xi_{2}\right), & s \prec \tau_{1}, s \prec \tau_{2}, \\ \psi_{s}\left(u_{1}-\xi_{1}, u_{2}\right), & s \prec \tau_{1}, s \succ \tau_{2}, \\ \psi_{s}\left(u_{1}, u_{2}-\xi_{2}\right), & s \succ \tau_{1}, s \prec \tau_{2}, \\ \psi_{s}\left(u_{1}, u_{2}\right), & s \succ \tau_{1}, s \succ \tau_{2} .\end{cases}
$$

Then one has the telescoping identity similar to (5.16):

$$
\Delta_{\xi_{1} \xi_{2}} \widehat{\Psi}_{t}=\sum_{\tau_{1}, \tau_{2} \succeq 0} \prod_{s \succeq 0} \theta_{\tau_{1} \tau_{2}}^{\xi_{1} \xi_{2}} \psi_{s}
$$

From (5.16), (5.17) we finally obtain

$$
\Delta_{\xi_{1} \xi_{2}} \partial_{12} \widehat{\Psi}_{t}=\sum_{\tau_{1}, \tau_{2} \succeq 0} \sum_{\sigma_{1}, \sigma_{2} \succeq 0} \prod_{s \succeq 0} \theta_{\tau_{1} \tau_{2}}^{\xi_{1} \xi_{2}} \vartheta_{\sigma_{1} \sigma_{2}} \psi_{s} .
$$

In (5.16)-(5.18), the sums are taken over all indices $\tau_{1}, \tau_{2}, \sigma_{1}, \sigma_{2} \in \mathbb{Z}^{d}$, respectively, which are completely ordered by the relation $\prec$, and the convergence of these series can be shown similarly as in [7]. From (5.14), (5.18) we obtain

$$
\left|q\left(z_{1}\right) q\left(z_{2}\right) z_{1} z_{2} \Psi_{t}\left(z_{1}, z_{2}\right)\right| \leqslant C \sum_{\tau_{1}, \tau_{2} \succeq 0} \sum_{\sigma_{1}, \sigma_{2} \succeq 0} \Gamma_{\tau_{1} \tau_{2} \sigma_{1} \sigma_{2}},
$$


where

$$
\Gamma_{\tau_{1} \tau_{2} \sigma_{1} \sigma_{2}}:=\int_{\mathbb{R}^{2}} d u_{1} d u_{2} \int_{[0,1]^{2}} \prod_{s \succeq 0}\left|\theta_{\tau_{1}}^{\xi_{1} \xi_{2}} \vartheta_{2} \vartheta_{\sigma_{1} \sigma_{2}} \psi_{s}\right| \xi_{1}^{-1-\delta^{\prime}} \xi_{2}^{-1-\delta^{\prime}} d \xi_{1} d \xi_{2} .
$$

Write $\sum_{\tau_{1}, \tau_{2} \succeq 0} \sum_{\sigma_{1}, \sigma_{2} \succeq 0}=\sum_{i=1}^{4} \sum_{|T|=i}$, where the last sum is taken over all subsets $T:=\left\{\tau_{1}, \tau_{2}, \sigma_{1}, \sigma_{2}\right\} \subset \mathbb{Z}^{d}$ of cardinality $|T|=i$ depending on the number of distinct indices in $T$. Clearly it suffices to show (5.7) for each sum $\sum_{|T|=i} \Gamma_{\tau_{1} \tau_{2} \sigma_{1} \sigma_{2}}, i=1,2,3,4$. To avoid tedious computations, we discuss below the extreme cases $i=1$ and $i=4$ only.

Similarly to (5.9), one can show that for each $k \geqslant 0$ there is a constant $C<\infty$ independent of $T \subset \mathbb{Z}^{d},|T|=1, \ldots, 4$, and such that

$$
\prod_{s \notin T, s \neq 0, t}\left|\theta_{\tau_{1} \tau_{2} \xi_{2} \xi_{2}} \vartheta_{\sigma_{1} \sigma_{2}} \psi_{s}\right| \leqslant C\left(1+\left|u_{1}\right|+\left|u_{2}\right|\right)^{-k}, \quad u_{1}, u_{2} \in \mathbb{R} .
$$

Consider first the "diagonal" sum $\sum_{|T|=1}$, or the case $\tau_{1}=\tau_{2}=\sigma_{1}=\sigma_{2}=: \tau$. Then

$$
\begin{aligned}
\theta_{\tau \tau}^{\xi_{1} \xi_{2}} \vartheta_{\tau \tau} \psi_{\tau}\left(u_{1}, u_{2}\right)= & \Delta_{\xi_{1} \xi_{2}} \partial_{12} \psi_{\tau}\left(u_{1}, u_{2}\right) \\
= & b_{\tau} b_{\tau-t}\left(\phi^{\prime \prime}\left(u_{1} b_{\tau}+u_{2} b_{\tau-t}\right)-\phi^{\prime \prime}\left(\left(u_{1}-\xi_{1}\right) b_{\tau}+u_{2} b_{\tau-t}\right)\right. \\
& -\phi^{\prime \prime}\left(u_{1} b_{\tau}+\left(u_{2}-\xi_{2}\right) b_{\tau-t}\right)+\phi^{\prime \prime}\left(\left(u_{1}-\xi_{1}\right) b_{\tau}\right. \\
& \left.\left.+\left(u_{2}-\xi_{2}\right) b_{\tau-t}\right)\right) .
\end{aligned}
$$

This holds for $\tau=0$ or $t$, because $\partial_{12} \psi_{\tau}\left(u_{1}, u_{2}\right)=b_{\tau} b_{\tau-t} \phi^{\prime \prime}\left(u_{1} b_{\tau}+u_{2} b_{\tau-t}\right)$ for any $\tau \in \mathbb{Z}^{d}$. Next,

$$
\begin{aligned}
\mid \phi^{\prime \prime} & \left(u_{1} b_{\tau}+u_{2} b_{\tau-t}\right)-\phi^{\prime \prime}\left(\left(u_{1}-\xi_{1}\right) b_{\tau}+u_{2} b_{\tau-t}\right) \\
& -\phi^{\prime \prime}\left(u_{1} b_{\tau}+\left(u_{2}-\xi_{2}\right) b_{\tau-t}\right)+\phi^{\prime \prime}\left(\left(u_{1}-\xi_{1}\right) b_{\tau}+\left(u_{2}-\xi_{2}\right) b_{\tau-t}\right) \mid \\
= & \left|E \zeta^{2} \mathrm{e}^{\mathrm{i}\left(u_{1} b_{\tau}+u_{2} b_{\tau-t}\right) \zeta}\left(1-\mathrm{e}^{-\mathrm{i} \xi_{1} b_{\tau} \zeta}\right)\left(1-\mathrm{e}^{-\mathrm{i} \xi_{2} b_{\tau-t} \zeta}\right)\right| \\
\leqslant & E \zeta^{2}\left|\left(1-\mathrm{e}^{-\mathrm{i} \xi_{1} b_{\tau} \zeta}\right)\left(1-\mathrm{e}^{-\mathrm{i} \xi_{2} b_{\tau-t} \zeta}\right)\right| \\
\leqslant & E \zeta^{2}\left(1 \wedge\left|\xi_{1} b_{\tau} \zeta\right|\right)\left(1 \wedge\left|\xi_{2} b_{\tau-t} \zeta\right|\right) .
\end{aligned}
$$

By the inequality $1 \wedge|x| \leqslant|x|^{\delta / 2}(\forall x \in \mathbb{R}, \forall \delta \in(0,1)$, and the moment condition (1.7), from (5.22) and (5.23) we obtain $\left|\theta_{\tau \tau}^{\xi_{1} \xi_{2}} \vartheta_{\tau \tau} \psi_{\tau}\left(u_{1}, u_{2}\right)\right| \leqslant C\left|b_{\tau} b_{\tau-t}\right|^{1+\delta / 2}\left|\xi_{1}\right|^{\delta / 2}\left|\xi_{2}\right|^{\delta / 2}$, $C=E|\zeta|^{2+\delta}$.

Taking $0<\delta^{\prime}<\delta / 2$ in (5.20), the inequality $\Gamma_{\tau \tau \tau \tau} \leqslant C\left|b_{\tau} b_{\tau-t}\right|^{1+\delta / 2}$ follows, yielding

$$
\sum_{|T|=1} \Gamma_{\tau_{1} \tau_{2} \sigma_{1} \sigma_{2}} \leqslant C \sum_{\tau \in \mathbb{Z}^{d}}|\tau|_{+}^{-\beta(1+\delta / 2)}|\tau-t|_{+}^{-\beta(1+\delta / 2)} \leqslant C|t|^{d-(2+\delta) \beta} .
$$

Next, consider $\sum_{|T|=4}$, or $\tau_{1}, \tau_{2}, \sigma_{1}, \sigma_{2}$ being all different. Let $T \cap\{0, t\}=\emptyset$. Then

$$
\theta_{\tau_{1} \tau_{2}}^{\xi_{1} \xi_{2}} \vartheta_{\sigma_{1} \sigma_{2}} \psi_{s}= \begin{cases}b_{\sigma_{1}} \phi^{\prime}\left(u_{1} b_{\sigma_{1}}+u_{2} b_{\sigma_{1}-t}\right), & s=\sigma_{1} \succ \tau_{1}, \tau_{2}, \\ b_{\sigma_{2}-t} \phi^{\prime}\left(u_{1} b_{\sigma_{2}}+u_{2} b_{\sigma_{2}-t}\right), & s=\sigma_{2} \succ \tau_{1}, \tau_{2}, \\ \phi\left(u_{1} b_{\tau_{1}}+u_{2} b_{\tau_{1}-t}\right)-\phi\left(\left(u_{1}-\xi_{1}\right) b_{\tau_{1}}+u_{2} b_{\tau_{1}-t}\right), & s=\tau_{1} \succ \tau_{2}, \\ \phi\left(u_{1} b_{\tau_{2}}+u_{2} b_{\tau_{2}-t}\right)-\phi\left(u_{1} b_{\tau_{2}}+\left(u_{2}-\xi_{2}\right) b_{\tau_{2}-t}\right), & s=\tau_{2} \succ \tau_{1}\end{cases}
$$


(similar expressions for $\theta_{\tau_{1} \tau_{2}}^{\xi_{1} \xi_{2}} \vartheta_{\sigma_{1} \sigma_{2}} \psi_{s}$ hold in the remaining cases). Hence

$$
\left|\theta_{\tau_{1} \tau_{2}}^{\xi_{1} \xi_{2}} \vartheta_{\sigma_{1} \sigma_{2}} \psi_{s}\right| \leqslant C \begin{cases}\left|b_{\sigma_{1}}\right|\left(\left(\left|u_{1}\right|+1\right)\left|b_{\sigma_{1}}\right|+\left(\left|u_{2}\right|+1\right)\left|b_{\sigma_{1}-t}\right|\right), & s=\sigma_{1}, \\ \left|b_{\sigma_{2}-t}\right|\left(\left(\left|u_{1}\right|+1\right)\left|b_{\sigma_{2}}\right|+\left(\left|u_{2}\right|+1\right)\left|b_{\sigma_{2}-t}\right|\right), & s=\sigma_{2}, \\ \left|\xi_{1} b_{\tau_{1}}\right|\left(\left(\left|u_{1}\right|+1\right)\left|b_{\tau_{1}}\right|+\left(\left|u_{2}\right|+1\right)\left|b_{\tau_{1}-t}\right|\right), & s=\tau_{1}, \\ \left|\xi_{2} b_{\tau_{2}-t}\right|\left(\left(\left|u_{1}\right|+1\right)\left|b_{\tau_{2}}\right|+\left(\left|u_{2}\right|+1\right)\left|b_{\tau_{2}-t}\right|\right), & s=\tau_{2}, \\ \left|b_{-t}\right|, & s=0, \\ \left|b_{t}\right|, & s=t .\end{cases}
$$

Therefore, for $T \cap\{0, t\}=\emptyset,|T|=4$ we obtain by (5.20), (5.21)

$$
\begin{aligned}
\prod_{s \succeq 0}\left|\theta_{\tau_{1} \tau_{2}}^{\xi_{1} \xi_{2}} \vartheta_{\sigma_{1} \sigma_{2}} \psi_{s}\right| \leqslant & C\left|\xi_{1} \xi_{2}\right|\left|b_{t} b_{-t}\right|\left(1+\left|u_{1}\right|+\left|u_{2}\right|\right)^{-k} \\
& \times\left(b_{\sigma_{1}}^{2}+b_{\sigma_{1}-t}^{2}\right)\left(b_{\sigma_{2}}^{2}+b_{\sigma_{2}-t}^{2}\right)\left(b_{\tau_{1}}^{2}+b_{\tau_{1}-t}^{2}\right)\left(b_{\tau_{2}}^{2}+b_{\tau_{2}-t}^{2}\right)
\end{aligned}
$$

and

$$
\sum_{|T|=4 ; T \cap\{0, t\}=\varnothing} \Gamma_{\tau_{1} \tau_{2} \sigma_{1} \sigma_{2}} \leqslant C\left|b_{t} b_{-t}\right| \leqslant C|t|^{-2 \beta}=\mathrm{O}\left(|t|^{d-(2+\delta) \beta}\right)
$$

provided $\delta \beta \leqslant d$. Similar bounds for $\left|\theta_{\tau_{1} \tau_{2}}^{\xi_{1} \xi_{2}} \vartheta_{\sigma_{1} \sigma_{2}} \psi_{s}\right|$ hold in the case $T \cap\{0, t\} \neq \emptyset$. This proves (5.7) and Lemma 5.1, too.

\section{REFERENCES}

[1] S. Albeverio, S.A. Molchanov, D. Surgailis, Stratified structure of the Universe and Burgers' equation - a probabilistic approach, Probab. Theory Related Fields 100 (1994) 457-484.

[2] V.V. Anh, J.M. Angulo, M.D. Ruiz-Medina, Possible long-range dependence in fractional random fields, J. Statist. Plan. Inf. 80 (1999) 95-110.

[3] H. Dehling, M.S. Taqqu, The empirical process of some long-range dependent sequences with an application to U-statistics, Ann. Statist. 17 (1989) 1767-1783.

[4] M. Denker, Weak convergence in nonparametric statistics, in: R. Bhattacharya, M. Denker (Eds.), Asymptotic Statistics, Birkhäuser, 1990.

[5] R.L. Dobrushin, P. Major, Non-central limit theorems for non-linear functions of Gaussian fields, Z. Wahrsch. Verw. Geb. 50 (1979) 27-52.

[6] P. Doukhan, D. Surgailis, Functional central limit theorem for the empirical process of short memory linear processes, C. R. Acad. Sci. Paris Serie 1326 (1998) 87-92.

[7] L. Giraitis, H.L. Koul, D. Surgailis, Asymptotic normality of regression estimators with long memory errors, Statist. Probab. Lett. 29 (1996) 317-335.

[8] L. Giraitis, D. Surgailis, Central limit theorem for the empirical process of a linear sequence with long memory, J. Stat. Plan. Inf. 80 (1999) 81-93.

[9] H.C. Ho, T. Hsing, On the asymptotic expansion of the empirical process of long memory moving averages, Ann. Statist. 24 (1996) 992-1024.

[10] H.C. Ho, T. Hsing, Limit theorems for functionals of moving averages, Ann. Probab. 25 (1997) 1636-1669.

[11] A.V. Ivanov, N.N. Leonenko, Statistical Analysis of Random Fields, Kluwer, Dordrecht, 1989.

[12] H.L. Koul, Weighted Empiricals and Linear Models, in: IMS Lecture Notes-Monograph Series, Vol. 21, Hayward, CA, 1992. 
[13] H.L. Koul, K. Mukherjee, Asymptotics of R-, MD- and LAD-estimators in linear regression models with long range dependent errors, Probab. Theory Related Fields 95 (1993) 535553.

[14] H.L. Koul, D. Surgailis, Asymptotic expansion of M-estimators with long memory errors, Ann. Statist. 25 (1997) 818-850.

[15] H.L. Koul, D. Surgailis, Asymptotics of empirical processes of long memory moving averages with infinite variance, Stoch. Proc. Appl. 91 (2001) 309-336.

[16] N.N. Leonenko, Random Fields with Singular Spectrum, Kluwer, Dordrecht, 1999.

[17] N.N. Leonenko, W.A. Woyczynski, Parameter identification for singular random fields arising in Burgers' turbulence, J. Statist. Plan. Inf. 80 (1999) 1-14.

[18] D. Marinucci, Gaussian semiparametric estimation for random fields with singular spectrum, Preprint, 2001.

[19] D. Surgailis, W.A. Woyczynski, Scaling limits of solutions of Burgers' equation with singular Gaussian initial data, in: C. Houdré, V. Pérez-Abreu (Eds.), Chaos Expansions, Multiple Wiener-Itô Integrals and Their Applications, CRC Press, Boca Raton, 1994, pp. 145-162.

[20] M.S. Taqqu, Convergence of integrated processes of arbitrary Hermite rank, Z. Wahrsch. Verw. Geb. 50 (1979) 53-83. 